\title{
Numerical investigation on a transition prediction model *
}

\author{
Zhen-xu SUN (孙振旭) ${ }^{1}$, Xiao-li ZHAO (赵晓利) ${ }^{2}$, \\ Jing-jing SONG (宋婧婧) ${ }^{1}$, Te-zhuan DU (杜特专) ${ }^{3}$ \\ (1. State Key Laboratory for Turbulence and Complex Systems, College of Engineering, \\ Peking University, Beijing 100871, P. R. China; \\ 2. School of Aerospace, Tsinghua University, Beijing 100084, P. R. China; \\ 3. Institute of Mechanics, Academia Sinica, Beijing 100190, P. R. China)
}

(Communicated by Shi-qiang DAI)

\begin{abstract}
A new transition prediction model is introduced, which couples the intermittency effect into the turbulence transport equations and takes the characteristics of fluid transition into consideration to mimic the exact process of transition. Test cases include a two-dimensional incompressible plate and a two-dimensional NACA0012 airfoil. Performance of this transition model for incompressible flows is studied, with numerical results consistent to experimental data. The requirement of grid resolution for this transition model is also studied.
\end{abstract}

Key words transition, turbulent modeling, intermittency, grid resolution, skin friction

Chinese Library Classification O343.8

2000 Mathematics Subject Classification $\quad 74 \mathrm{~K} 20$

\section{Introduction}

One challenging work in computational fluid dynamics is to predict transition from laminar flow to turbulence correctly. A mature theory on transition has already been established recently, where three types of transitions are specified, namely the natural transition, bypass transition ${ }^{[1]}$, and separated transition ${ }^{[2]}$. Natural transition occurs with relatively low turbulence intensity, while bypass transition occurs with higher turbulence intensity than natural transition. Separated transition usually occurs along with a high adverse pressure gradient.

Locating the transition point is usually of particular interest to researchers. In engineering applications, empirical formulas such as the Granville method, Michel method, etc. are widely utilized for simplicity. However, these methods are always extracted from a large amount of experimental data, which also becomes a main deficiency for the wide application. With the development of modern computers, classical approaches, such as the large eddy simulation (LES), the direct numerical simulation (DNS), and the stability analysis theory, are put into practice. The first two methods can solve Navier-Stokes (NS) equations and give a detailed description of fluctuations. However, they would become prohibitively expensive at high Reynolds numbers

* Received May 5, 2009 / Revised Oct. 26, 2009

Project supported by the National Science and Technology Pillar Program during the Eleventh FiveYear Plan Period (No. 2009BAG12A01)

Corresponding author Te-zhuan DU, Ph. D., E-mail: dutezhuan@imech.ac.cn 
due to a heavy mesh requirement of the amount of $R e^{9 / 4}$ (for DNS especially). Two basic ways are performed in the stability analysis theory, namely, the parabolic stability equations approach and the $e^{N}$ approach ${ }^{[3-4]}$. The latter is widely applied in modern engineering based on a linear stability theory. It assumes that the initial disturbance propagates downstream with a constant frequency, and the transition is completed when the amplitude of the disturbance amplifies to the $e^{N}$ order of its origin. Another widely used approach is the Reynolds average Navier-Stoke equations (RANS) model, which can give a statistical solution to turbulence. By doing Reynolds average on NS equations, transport equations for the Reynolds stress can be extracted, which is a description of turbulent quantities' evolution. Less computation cost is achieved because of its less stringentness on mesh requirement. However, the RANS models aim to solve a fully turbulent flow, which brings an earlier transition and a shorter transition zone when they are used for transition predictions. Amendments on the RANS models have been performed by several researchers. In order to predict the transition with high Reynolds numbers, Zheng et al. ${ }^{[5]}$ exerted the total stresses limitation (TSL) directly on RANS transport equations and attained good results. Further research reveals that the conventional RANS models approach takes no consideration of the flow's physical characteristics and only predicts transitions at the statistical level. These sheds light on a new approach taking the intermittency into consideration.

Flows in the transition zone keep laminar and turbulent alternately due to turbulent spots. The intermittency factor $\gamma$ is introduced to describe this phenomenon, which is defined as the ratio of the time of turbulence and the time of laminar flow in a period. $\gamma$ equals 1 with a fully developed turbulence and 0 with a laminar flow. By embedding an intermittency factor with turbulence models, better approaches to predicting transition can be obtained. In 1958, an intermittency factor coupled with the RANS models was introduced by Dhawan and Narasimha ${ }^{[6]}$, who assumed flow as a mixture of laminar flow and turbulence weighted by the intermittency factor. In 1992, a new $k-\epsilon-\gamma$ model coupling $\gamma$ with the $k$ - $\epsilon$ turbulence model was utilized by Cho and Chung ${ }^{[7]}$, which is effective in solving free shear layer problems such as planar jet flows and circle jet flows. In 2006, Menter ${ }^{[8]}$ invented a new transition prediction model based on local variables. He obtained an empirical formula on $R e_{\theta_{t}}$ by numerical experiments, established a transport equation for intermittency factor, and succeeded in predicting transition in three-dimensional flow fields. Researchers in China have also carried out related studies and obtained several promising achievements. In 2009, a new $k-\omega-\gamma$ model was introduced by Wang and $\mathrm{Fu}^{[9]}$, succeeding in predicting boundary transitions in subsonic, supersonic, and hypersonic fields.

In the present work, an $R_{t}-\gamma$ transition prediction model ${ }^{[10]}$ is adopted, which is a brand new model embedding transport equations of intermittency factor with the one-equation $R_{t}$ model ${ }^{[11]}$. Compared with the $k-\epsilon-\gamma$ and $k-\omega-\gamma$ models, it is simpler because of the fewer transport equations and thus reduces the computational cost. In order to carry out the research on this model, the two-dimensional planar boundary and the two-dimensional NACA0012 airfoil are chosen as test cases compared to corresponding experimental data. The results reveal that it is an excellent transition prediction model with high efficiency and accuracy.

\section{$1 \quad R_{t}-\gamma$ transition model}

\subsection{Governing equations}

The fluid under consideration is an incompressible viscous flow. The primary transport variables of the fluid field are the velocity $U$ and the fluid pressure $P$. These variables are governed by conservation equations of mass and momentum:

$$
\frac{D \rho}{D t}=0
$$




$$
\frac{D U_{i}}{D t}+\frac{1}{\rho} \frac{\partial P}{\partial x_{i}}=-\frac{\partial \tau_{i j}}{\partial x_{j}}+\frac{\partial}{\partial x_{j}}\left(\nu \frac{\partial U_{i}}{\partial x_{j}}\right) .
$$

The one-equation $R_{t}$ model is a linear viscosity model ${ }^{[12]}$ so that the Boussinesq approximation is adopted. Thus, the Reynolds stresses are expressed as

$$
\tau_{i j}=\frac{2}{3} k \delta_{i j}-2 v_{t} S_{i j}
$$

where $k$ is the turbulent kinetic energy, $k=\frac{1}{2} \tau_{i i}$. The isotropic eddy viscosity is a consequence of the Boussinesq approximation, which assumes a direct proportionality between the turbulent Reynolds stress and the mean strain rate field. Therefore, the most important part is how to express the turbulent eddy viscosity $\mu_{t}$. In the $R_{t}-\gamma$ model, the undamped eddy viscosity $R$ is introduced to relate $\mu_{t}$ in the form: $\mu_{t}=f_{\mu} \rho R$, where $f_{\mu}$ is a damping function taking the form

$$
f_{\mu}=\frac{\tanh \left(\alpha\left(R /\left(C_{\mu} v\right)\right)^{2}\right)}{\tanh \left(\beta\left(R /\left(C_{\mu} v\right)\right)^{2}\right)}
$$

The transport equation of the undamped eddy viscosity is given as

$$
\frac{D(\rho R)}{D t}=\frac{\partial}{\partial x_{j}}\left[\left(\mu+\frac{\mu_{t}}{\sigma_{r}}\right) \frac{\partial R}{\partial x_{j}}\right]+\rho\left(C_{1}-C_{2} f_{2} f_{r}\right) \sqrt{R P_{k}}-\rho C_{3} D .
$$

Here, the turbulent transport term and the pressure diffusion term have already been related with the undamped eddy viscosity $R$ by the coefficient $-\mu_{t} / \sigma_{r}$. The first term on the righthand side is called the molecular diffusion term, and represents the diffusion of the turbulence energy caused by the molecular transport process. The third term on the right-hand side is the production term, and is regarded as the rate at which the undamped eddy viscosity is transferred from the mean flow to the turbulence. It is defined as

$$
P_{k}=v_{t}\left[\left(\frac{\partial U_{i}}{\partial x_{j}}+\frac{\partial U_{j}}{\partial x_{i}}\right) \frac{\partial U_{i}}{\partial x_{j}}-\frac{2}{3}\left(\frac{U_{k}}{x_{k}}\right)^{2}\right] .
$$

Here, the turbulent kinetic viscosity is expressed as a function of the mean strain rate. The last term on the right-hand side is the dissipation term $D$, which is the rate at which the undamped viscosity is converted into the thermal internal energy. Hence, the quantity $D$ is given by

$$
D=\left\{\begin{array}{l}
\frac{\partial R}{\partial x_{j}} \frac{\partial R}{\partial x_{j}}, \quad \frac{\partial Q}{\partial x_{j}} \frac{\partial R}{\partial x_{j}}>0, \\
0, \quad \frac{\partial Q}{\partial x_{j}} \frac{\partial R}{\partial x_{j}} \leq 0
\end{array}\right.
$$

The transport equation for the intermittency factor is assumed to have the following form:

$$
\begin{aligned}
\frac{D(\rho \gamma)}{D t}= & \frac{\partial}{\partial x_{j}}\left\{\left[\mu+(1-\gamma) \frac{\mu_{t}}{\sigma_{r}}\right] \frac{\partial \gamma}{\partial x_{j}}\right\} \\
& +\rho \gamma(1-\gamma) \sqrt{\frac{C_{\mu} P_{k}}{R}}\left(C_{\gamma 1}-C_{\gamma 2} \Gamma\right)+C_{\gamma 3} \mu_{t} \frac{\partial \gamma}{\partial x_{j}} \frac{\partial \gamma}{\partial x_{j}}
\end{aligned}
$$

where

$$
\Gamma=\frac{\left(R / C_{\mu}\right)^{4 / 5}}{P_{k}^{3 / 4}} \frac{U_{i}}{Q} \frac{\partial U_{i}}{\partial x_{j}} \frac{\partial \gamma}{\partial x_{j}}, \quad Q=\sqrt{U_{l} U_{l}}
$$


Closure coefficients in the model are listed as

$$
\begin{aligned}
& \alpha=7.2 \times 10^{-4}, \quad \beta=0.2, \quad C_{\gamma 1}=1.6, \quad C_{\gamma 2}=0.16, \\
& C_{\gamma 3}=0.15, \quad \sigma_{R}=\sigma_{r}=1.0, \quad C_{\mu}=0.09, \quad \kappa=0.41, \\
& C_{1}=C_{2}+\frac{\kappa^{2}}{2 \sigma_{R}}, \quad C_{2}=\frac{12}{11} \sqrt{\frac{\beta}{\alpha}}, \quad C_{3}=\frac{3}{2 \sigma_{R}} .
\end{aligned}
$$

\subsection{Algorithms}

For subsonic flows, the conventional Riemann solver ${ }^{[13]}$ is utilized in the spatial discretization, and the second order total variation diminishing (TVD) scheme with a minmod limiter is also used. For the temporal discretization, a point-implicit scheme is used. It tends to slow the convergence when using the conventional Riemann solver to deal with subsonic flows in a compressible way. In order to eliminate this ill-effect, a preconditioning approach ${ }^{[14-17]}$ is required. Preconditioning amends the time evolution by altering the signal propagation speed calculated by the eigenvalues of the Jacobian matrices.

In order to make a comparison with experiments, a series of T3 experimental data has been adopted in this paper. For comparison, the skin friction is selected as the indicator of transition, which is a significant criterion to depict transition. Early in the 20th century it has been studied by Blasius for his doctoral dissertation. A Blasius solution was given through solving the laminar boundary layer equations. From then on, several researchers also derived some empirical formulas to revise the Blasius solution. Therefore, a detailed study can be carried out by comparing skin friction.

There is a great difference in the aerodynamic properties with or without transition for flying objects. Therefore, the research on drag coefficients and pressure coefficients of an NACA0012 airfoil with incidence is performed below by using the $R_{t}-\gamma$ model and a non-transitional realizable $k$ - $\epsilon$ model.

\section{Test cases and results}

\subsection{Incompressible flat-plate boundary layer}

In order to test the efficiency of the $R_{t^{-}} \gamma$ model, an incompressible flat-plate boundary layer is chosen. Experimental data comes from the experimental results carried out by researchers at Rolls-Royce ${ }^{[18]}$. Meanwhile, a comparison is done with Menter's computational results ${ }^{[8]}$. The space coordinate is $\boldsymbol{x}=(x, y)$, where $x$ corresponds to the stream-wise direction and $y$ the cross-stream direction. The fluid in this study is the air at room temperature. Computations are performed on a domain of $[0,0.25 \mathrm{~m}] \times[0,0.04 \mathrm{~m}]$. Constant pressure, temperature, and velocity are imposed to the inlet and upper boundaries. Setting the free flow Mach number $M a=0.2$, simulations are performed for two turbulence intensities, $3.5 \%$ and $6.5 \%$, named $\mathrm{T} 3 \mathrm{~A}$ and $\mathrm{T} 3 \mathrm{~B}$, respectively.

\subsubsection{T3A case}

The distribution of the skin friction along the flat-plate is shown in Fig. 1, in which the Mach number and the turbulence intensity are 0.2 and $3.5 \%$, respectively. The $x$-coordinate in Fig. 1 refers to the local Reynolds number $\left(\times 10^{6}\right)$ given by

$$
R e_{x}=\frac{U \cdot x}{v} .
$$

An analysis of the sharp increase in the skin friction suggests that, after the transition, fluid quantities such as mass, momentum, and energy begin to exchange significantly between macro fluid particles through fluctuations, which leads to the diffusion of turbulence. The turbulent skin friction and thermal exchanges become much stronger compared with the diffusion caused by molecular transports, which means a sudden increase in the skin friction in the fully turbulent 
zone. The minimal skin friction computed by the $R_{t}-\gamma$ model locates nearby $R e_{x}=1.8 \times 10^{6}$, which is almost in good accordance with experimental data, indicating an excellent prediction of the transition point. The last part of the profile simulated by the $R_{t}-\gamma$ model also shows a good agreement with the experimental points. The deficiency of this model is that there is a much steeper distribution of the transition zone than that in the experiment, which maybe is a consequence of bypass transition prediction in the description hereafter.

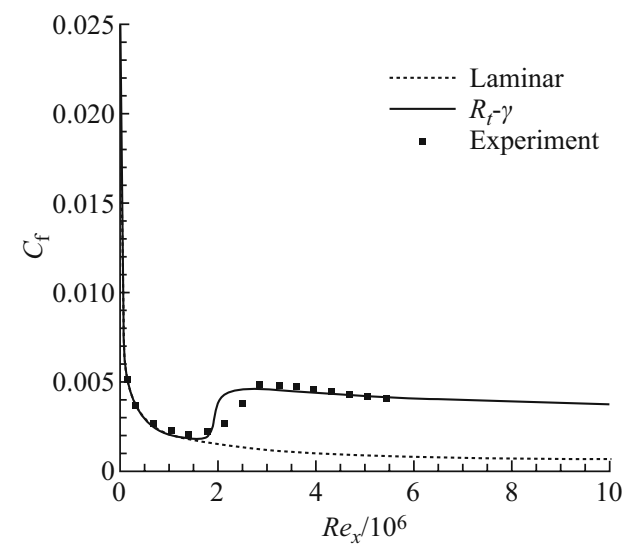

Fig. 1 Distribution of skin friction $\left(C_{\mathrm{f}}\right)$ along the flat-plate with $I=3.5 \%$

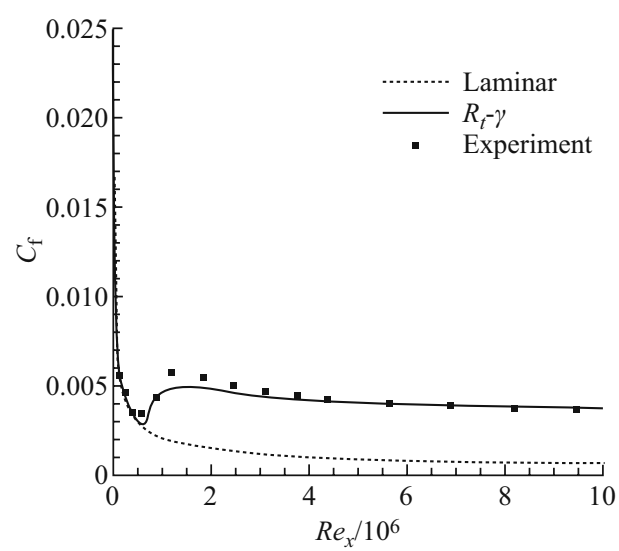

Fig. 2 Distribution of skin friction $\left(C_{\mathrm{f}}\right)$ along the flat-plate with $I=6.5 \%$

\subsubsection{T3B case}

Figure 2 shows the result of simulations with the turbulence intensity of $6.5 \%$. Compared with the T3A case, this is more like a kind of bypass transition. Menter's model shows a much earlier transition and a relatively big difference between his minimal skin friction and experimental data. Moreover, slightly higher values can be seen in his results even in the fully turbulent zone compared with experimental results. However, results using the $R_{t}-\gamma$ model are basically consistent with the experiment, no matter the length of the transition zone or the extreme points in the curve. The reason is probably related with the transition type, i.e., bypass transition. The length of the transition zone decreases as the turbulence intensity grows bigger, indicating a better capability for the $R_{t}-\gamma$ model to predict the bypass transition. It also can be seen from the turbulent zone that the $R_{t}-\gamma$ model shows a better prediction than Menter's model.

\subsubsection{Effects of grid resolution}

Effects of grid resolution can be examined by numerical simulations on grids with different orders of near-wall distance. In this part, orders of $O(10), O(1)$, and $O(0.1)$ have been tried, and the results of these simulations are compared. For the case of $O(10)$, the simulation is performed with various near-wall treatments, such as wall functions and directly solving. The T3A and T3B cases are both examined, and all the settings are kept the same with the previous study.

When $y^{+}$is of the order of 10 , the results in both cases show a great difference with experiments, which indicates an unreasonable simulation as the dash-dot lines shown in Fig. 3 and Fig. 4. One reason can be sought. As the grid grows coarse, the diffusion caused by the coarse grid becomes larger, and acts as a numerical dissipation, which can be confused easily with the dissipation caused by turbulence. As a result, an earlier transition happens and higher values of skin friction are observed. The solid lines and the dashed lines in Fig. 3 and Fig. 4 indicate that the good agreement with experiments can be attained as long as $y^{+}$is less than or equal 


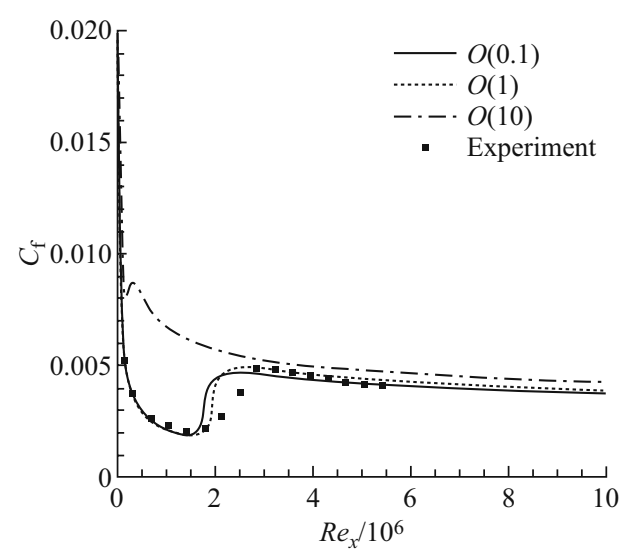

Fig. 3 Surface distribution of skin friction $\left(C_{\mathrm{f}}\right)$ along the flat-plate in T3A case

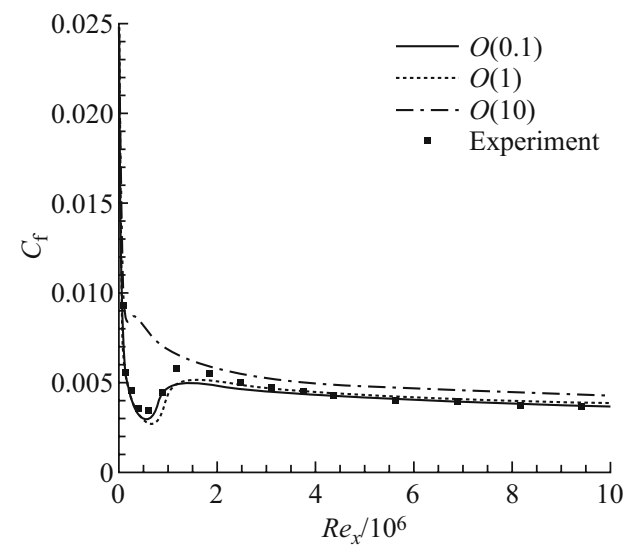

Fig. 4 Surface distribution of skin friction $\left(C_{\mathrm{f}}\right)$ along the flat-plate in T3B case

to the order of 1 . This can be deduced from the definition of skin friction that is linear with the gradient of velocity $U$ over $y$, the cross-stream direction. The profile of velocity along the cross-stream direction keeps linear with $y$ when $y^{+}$is less than 5 , which means the skin friction stays constant as long as $y^{+}$is less than or equal to the order of 1 . The above analysis reveals that the grids resolution with the order of 1 is sufficient to predict transition, and there is no need to mesh the grid much finer. Moreover, a coarser grid with an order of 10 or larger should be avoided due to the ignorance of near-wall turbulent behaviors. The wall function approach assumes a statistical behavior of velocity, which is not suitable for transition prediction.

\subsection{Analysis of two-dimensional NACA0012 airfoil}

As a fairly typical type of airfoil, NACA0012 has been widely used for helicopter rotor blades for a considerable number of years, and its stalling behavior under a certain angle of attack is still of particular interest to researchers. Transition always occurs when fluid passes through the airfoil due to an adverse pressure gradient, which leads to a sudden change in aerodynamic properties. In this section, in order to better study the airfoil's aerodynamic properties, different turbulent models are utilized. An ordinary realizable $k-\epsilon$ model is used here to make a comparison with the $R_{t}-\gamma$ transition model.

The airfoil in this simulation is of $0.76 \mathrm{~m}$ chord. The numerical simulation is performed with a free stream $M a=0.16, R e=2.88 \times 10^{6}, U=55 \mathrm{~m} / \mathrm{s}$, under various angles of attack, i.e., $0^{\circ}, 2^{\circ}, 4^{\circ}, 6^{\circ}, 8^{\circ}$, and $10^{\circ}$, respectively. Drag coefficients and surface pressure coefficients are chosen as the main concerned properties, compared between different turbulent models and experimental data. The grid used is presented as Fig. 5.

2.2.1 Comparison of surface pressure coefficient $\left(C_{\mathrm{p}}\right)$

As long as the pressure distribution along the airfoil is known, the aerodynamic characteristics of the airfoil can be deduced in a simple way. The surface pressure coefficient, given by $C_{\mathrm{p}}=\frac{2\left(P-P_{\infty}\right)}{\rho U_{\infty}^{2}}$, become a particular factor when a study on the airfoil is carried out. Transitions always occur due to a large adverse pressure gradient when the airfoil flies with a big incidence. Special attention should be paid to that when a numerical simulation is performed, turbulent models with or without transition prediction could lead to considerable difference in aerodynamic properties on the surface of the airfoil. Unreasonable results can be obtained if the transition is not taken into consideration.

Simulations on the two-dimensional NACA0012 airfoil with various pitch angles are performed and the profiles of $C_{\mathrm{p}}$ along the upper surface are sketched in Figs. 6(a)-6(f), with the 


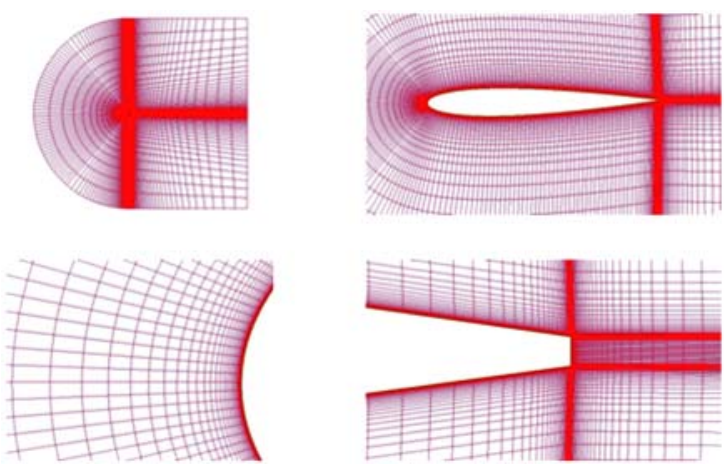

Fig. 5 Grid in the simulation of NACA0012 airfoil

results from different turbulent models and the experimental data from a fundamental study on the NACA0012 airfoil by Gregory and O'Reilly ${ }^{[19]}$. It can be seen from these figures that simulations with a transitional model always agree well with the experimental data, while simulations with the conventional realizable $k-\epsilon$ model always give a worse prediction, which can be related with that the inner physics of fluid can be perfectly captured by the $R_{t}-\gamma$ transition prediction model rather than the conventional realizable model. Another simulation is performed by embedding a leading-edge transition model with the conventional realizable $k-\epsilon$ model. The results show that a prediction of $C_{\mathrm{p}}$ is obtained as well as that with the $R_{t}-\gamma$ model, except for a relatively long CPU time. As the angle of attack increases, the effects of transition become dominant, announcing a failure application of the conventional realizable $k-\epsilon$ model. The results shown in Fig. 6(e) reveal that the $R_{t}-\gamma$ model acts significantly better in a big angle of attack whilst the realizable $k-\epsilon$ model gives a totally ridiculous result. The accuracy for the $R_{t^{-}} \gamma$ model increases as the angle of attack increases.

\subsubsection{Drag coefficient $\left(C_{\mathrm{d}}\right)$}

As a particularly important non-dimensional variable, the drag coefficient is defined by $C_{\mathrm{d}}=\frac{2 F_{\mathrm{d}}}{\rho U_{\infty}^{2}}$, where $F_{\mathrm{d}}$ is the drag. Simulations under different angles of attack are performed to obtain $C_{\mathrm{d}}$ for different turbulent models. Compared to the experimental results in [19], the $R_{t}-\gamma$ model shows an acceptable similarity, whilst the realizable $k$ - $\epsilon$ model fails to predict drag coefficients correctly, especially when the angle of attack increases over $5^{\circ}$. This can be attributed to the instability that is a result of the vortex shedding in the wake of the airfoil. Figure 7 also indicates that a transitional turbulence model should be utilized as long as the flow experiences a transition and the $R_{t}-\gamma$ model operates well as a transition prediction model.

\section{Concluding remarks}

In the present paper, an $R_{t^{-}} \gamma$ model based on the one-equation turbulent $R_{t}$ model is discussed, and numerical simulations in subsonic applications with this model are performed. With local variables used, the $R_{t}-\gamma$ model benefits in a low computational cost and fast convergence. Because of the relatively deficient data from three-dimensional large scale complicated experiments, data from a two-dimensional flat-plate boundary layer experiment and a two-dimensional NACA0012 airfoil experiment have been adopted here. Numerical results show that this model can correctly predict the fluid's behavior through and after the transition zone. A perfect agreement with experimental data is achieved, indicating that the $R_{t}-\gamma$ model is a promising transition prediction model with high accuracy and is very suitable for flows with high Reynolds numbers. The analysis of grid resolution reveals that a near-wall distance requirement of $y^{+} \leq 1$ 


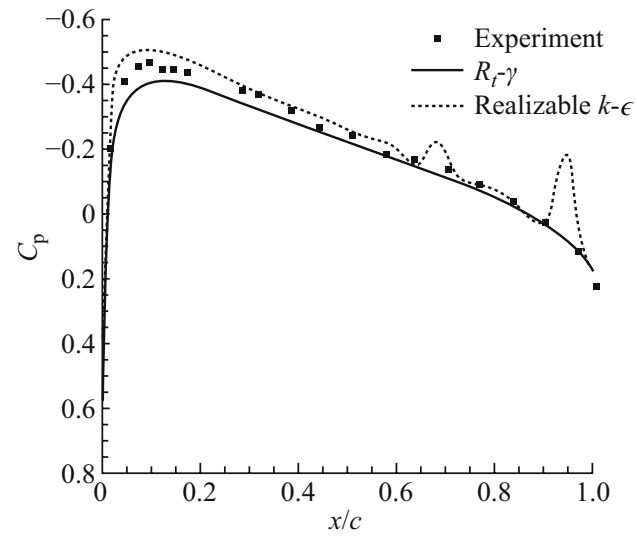

(a) The angle of attack is $0^{\circ}$

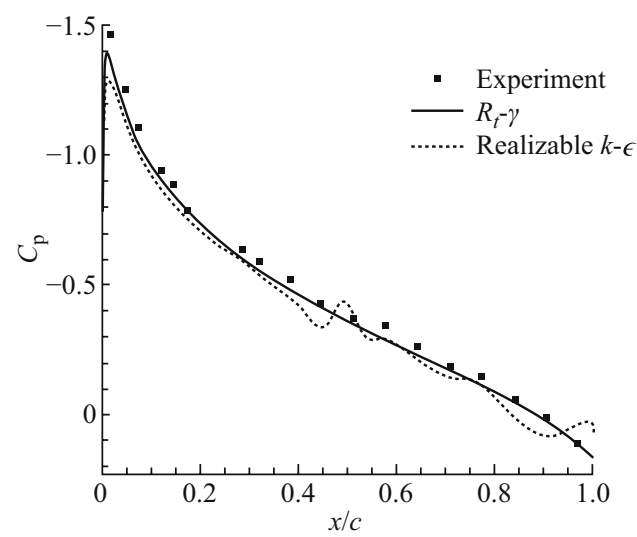

(c) The angle of attack is $4^{\circ}$

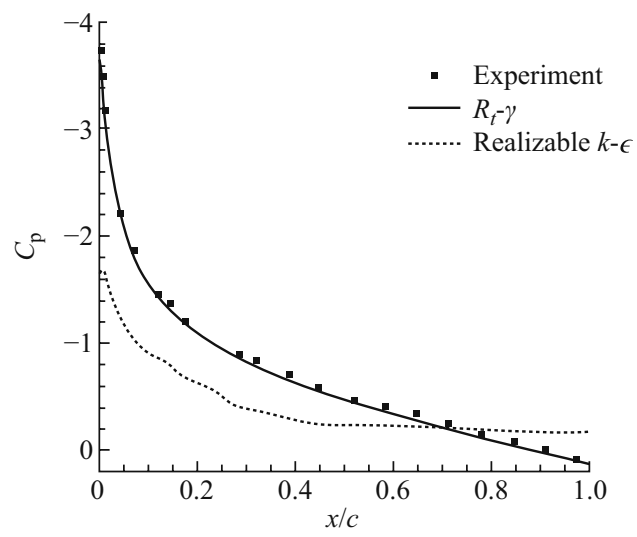

(e) The angle of attack is $8^{\circ}$

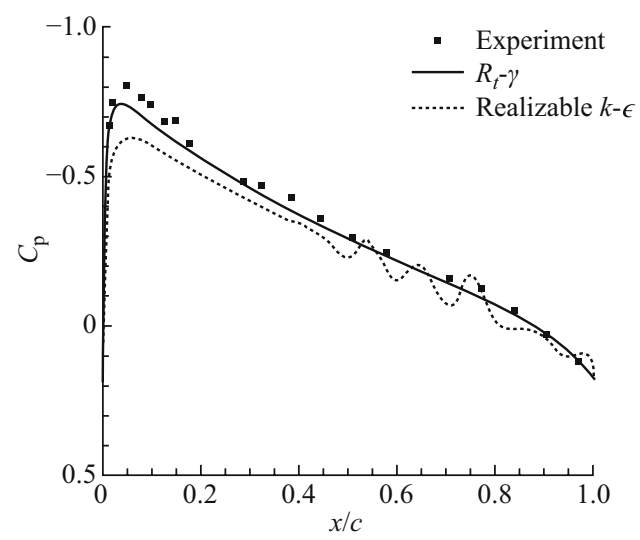

(b) The angle of attack is $2^{\circ}$

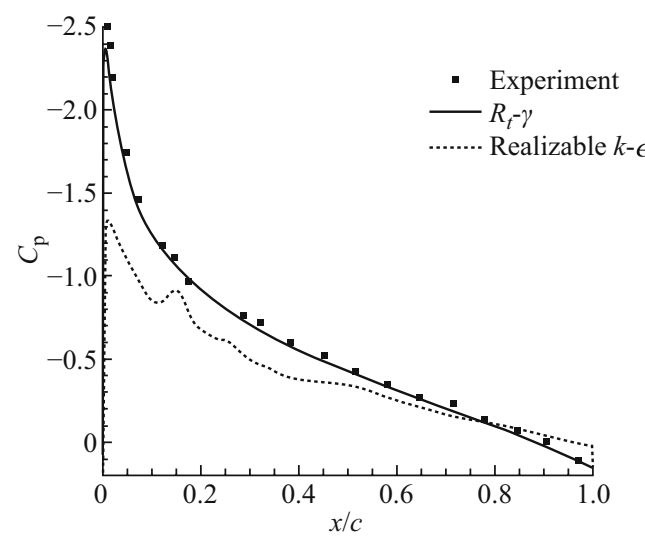

(b) The angle of attack is $6^{\circ}$

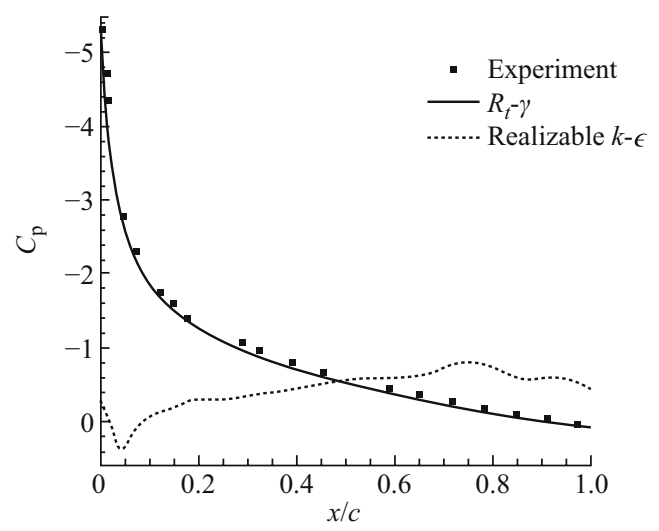

(f) The angle of attack is $10^{\circ}$

Fig. 6 Pressure distribution on the upper surface of NACA0012 airfoil

is needed to correctly predict transition.

Predicting transition still remains a fairly complicated problem, and a lot of difficulties exist when numerical simulations are performed. Initial values of the turbulent variables should be given when using a turbulent model, while in the present study it is done by giving the free 


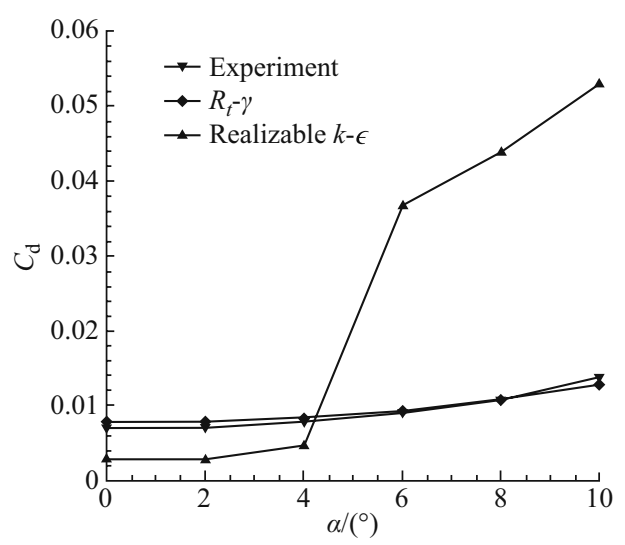

Fig. $7 \quad C_{\mathrm{d}}$ under different pitch angles

stream turbulence intensity and a ratio of molecular viscosity and turbulent eddy viscosity. The latter is a sensitive factor. It is an equivalent quantity to turbulence length scale, which is usually pretty difficult to estimate.

The transition in supersonic and hypersonic fields is still a key problem for researchers today. Because of the advantages of the one-equation $R_{t}$ model in supersonic and hypersonic fields, a promising application prospect of the $R_{t}-\gamma$ model in these fields can be foreseen, which will be continuously studied in further research.

Acknowledgements The authors would like to thank Professor Chen, Yao-song and Professor An, Yi-ran for their great support and precious advice for this paper.

\section{References}

[1] Jacobs, R. G. and Durbin, P. A. Simulations of bypass transition. Journal of Fluid Mechanics 428, 185-212 (2001)

[2] Mayle, R. E. Transition in a separation bubble. ASME Journal of Turbomachinery 118(4), 752759 (1996)

[3] Cebeci, T. and Stewartson, K. On stability and transition in three-dimensional flows. AIAA Journal 18(4), 398-405 (1997)

[4] Mack, L. M. Stability of three dimensional boundary layers on swept wings at transonic speeds. Proc. IUTAM Symposium III (eds. Zierep, J. and Oertel, H.), Springer-Verlag, Toulouse, 209-224 (1989)

[5] Zheng, X. Q., Liu, C. Q., Liu, F., and Yang, C-I. Turbulent transition simulation using the $k-\omega$ model. International Journal for Numerical Methods in Engineering 42(5), 907-926 (1998)

[6] Dhawan, S. and Narasimha, R. Some properties of boundary-layer flow during transition from laminar to turbulent motion. Journal of Fluid Mechanics 3(4), 414-436 (1958)

[7] Cho, J. R. and Chung, M. K. A $k-\epsilon-\gamma$ equation turbulence model. Journal of Fluid Mechanics 237, 301-322 (1992)

[8] Menter, F. R. Transition modeling for general purpose CFD codes. Flow Turbulence Combust 77(1-4), 277-303 (2006)

[9] Wang, Liang and Fu, Song. A new transition/turbulence model for the flow transition in supersonic boundary layer (in Chinese). Chinese Journal of Theoretical and Applied Mechanics 41(2), 162168 (2009)

[10] CFD++ User Manual, Version 8.1, Metacomp Technologies, Agoura Hills, CA. 12 (2009) 
[11] Goldberg, U. Turbulence closure with a topography-parameter-free single equation model. Int. J. CFD 17(1), 27-38 (2003)

[12] Launder, B. E. Closure Strategies for Turbulent and Transitional Flows, Cambridge University Press, 9-46 (2002)

[13] Batten, P., Leschziner, M. A., and Goldberg, U. C. Average-state Jacobians and implicit methods for compressible viscous and turbulent flows. Journal of Computational Physics 137(1), 38-78 (1997)

[14] Chorin, A. J. A numerical method for solving incompressible viscous flow problems. Journal of Computational Physics 135(2), 118-125 (1997)

[15] Leer, B. Van, Lee, W.-T., and Roe, P. L. Characteristic time-stepping or local preconditioning of the Euler equations. AIAA Computational Fluid Dynamics Conference, 10th, Honolulu, HI, June 24-27, 1991, Technical Papers A91-40701, 17-34; American Institute of Aeronautics and Astronautics, Washington, D. C., 260-282 (1991)

[16] Weiss, J. M. and Smith, W. A. Preconditioning applied to variable and constant density flows. AIAA Journal 33(11), 2050-2057 (1995)

[17] Vingeron, D., Deliege, G., and Essers, J. A. Low Mach number local preconditioning for unsteady viscous finite volumes simulations on 3D unstructured grids. European Conference on Computational Fluid Dynamics (ECCOMAS CFD 2006), TU Delft, The Netherlands (2006)

[18] Savill, A. M. By-pass transition using conventional closures. Closure Strategies for Turbulent and Transitional Flows (eds. Launder, B. E. and Sandham, N. D.), Cambridge University Press, Cambridge, Ch. 17, 464-492 (2002)

[19] Gregory, N. and O'Reilly, C. L. Low-Speed Aerodynamic Characteristics of NACA0012 Airfoil Section, Including the Effects of Upper-Surface Roughness Simulating Hoar Frost, TR, NPL AERO Rept. 1308, Middlesex, England, UK (1970) 